\title{
A Presuppositional Account of Proportional Ambiguity
}

\author{
Chris Barker \\ Center for Cognitive Science, Ohio State University
}

It has been well-known at least since Partee (1984) that there is a systematic ambiguity associated with proportional adverbial quantifiers such as usually and mostly, and ever since Kadmon (1987) this ambiguity has been known as 'the proportion problem'. Most people who have studied the proportion problem have concentrated primarily on describing the range of truth conditions associated with proportional sentences. This has turned out to be a delicate and complicated problem, and the issues it raises have by no means been settled in the literature yet. However, I would like to concentrate on a slightly different aspect of the proportion problem. One of the peculiar things about proportional sentences is that one reading often seems to be strongly preferred and the other expected readings are either difficult or impossible to get. This paper, then, sets out to explore the factors that constrain which of several construals of a proportional sentence will be available for a token uttered in a particular context. I will propose that there is a conventional implicature associated with the use of a proportional adverbial quantifier, and that different proportional readings give rise to distinct presuppositions. On this view, sentences involving a proportional adverbial quantifier are indeed ambiguous across a number of distinct interpretations, but only some interpretations will be felicitous on a given occasion of use.

\section{Truth conditions for proportional adverbial quantifiers}

In order to make the pragmatic analysis presented below explicit and concrete, it will be necessary to describe the truth conditions of proportional sentences with some precision. For the sake of simplicity, I will adopt a refinement of Lewis' (1975) so-called 'unselective binding' approach to adverbial quantification; however, nothing crucial hinges on this choice. In particular, I see no obstacle to reconstructing the pragmatic analyses developed here in a dynamic framework (cf. the discussions of the proportion problem in e.g. de Swart (1991) or Chierchia (1992)).

(1) a. Usually, if a woman owns a dog, she is happy.

b. Usually, if an artist lives in a town, it is pretty.

c. Usually, if a semanticist hears of a good job, she applies for it.

The sentences in (1) all share certain structural similarities: an adverbial quantifier whose restriction is characterized by an if clause containing two indefinites and whose nuclear scope is characterized by the main clause. 
However, the most natural interpretations of these sentences have nonparallel truth conditions as suggested by the rough paraphrases in (2).

(2) a. Most women who own at least one dog are happy.

b. Most towns which contain at least one artist are pretty.

c. Most semanticist/good-job hearings lead to applications.

To adopt the descriptive terminology of Kadmon (1987), we can say that (1a) involves asymmetric quantification over the first indefinite; (1b) involves asymmetric quantification over the second indefinite; and (1c) involves symmetric quantification over both of the indefinites simultaneously. That is, we can distinguish the three readings in (1) by noting whether the quantifier "binds" the first indefinite, the second, or both.

To do this, we will need to keep track of two kinds of variables, primed and unprimed. Therefore I will introduce the following notational conventions:

(3) $E=$ a set of entities

$=\left\{d_{1}, d_{2}, \ldots, j_{1}, j_{2}, \ldots, s_{1}, s_{2}, \ldots, w_{1}, w_{2}, \ldots\right\}$

$N=$ a set of entity-denoting variables, primed and unprimed

$=N_{0} \cup N^{\prime}=\{x, y, \ldots\} \cup\left\{x^{\prime}, y^{\prime}, \ldots\right\}$

$F=$ a set of partial assignment functions from subsets of $N$ into $E$ $=\left\{f, f_{a}, f_{b}, \ldots, g, g_{a}, g_{b}, \ldots\right\}$

This logical vocabulary allows translations of the sentences in (1) which are disambiguated in the relevant respect as given in (4). Intuitively, the primed variables will be those variables which are dominant in the quantification.

Quantifier(Restriction, Nuclear ScOPE)

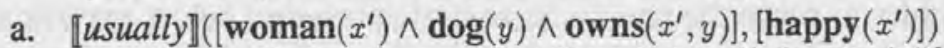

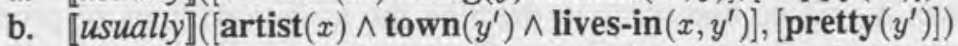

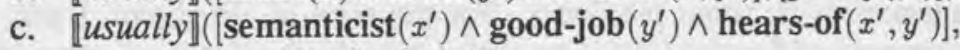
[applies-for $\left.\left(x^{\prime}, y^{\prime}\right)\right]$ )

Note that I have given translations of the restrictions and the nuclear scopes in (9) as formulas containing free variables in the style of Heim's (1982: chapter II) elaboration of Lewis' basic approach. Unselective binding is notoriously inadequate for distinguishing proportional readings. But as pointed out in Root (1985) and elsewhere, this deficiency can be easily remedied by replacing Lewis' original conception of a quantificational case as corresponding to an individual assignment function with the notion of an equivalence class of assignment functions: 
(5) Inducing a partition on the set of assignment functions:

Two assignment functions $f$ and $g$ are members of the same equivalence class (i.e. the same quantificational CASE)

iff they agree on what they assign to all primed variables:

$$
\begin{gathered}
{[\operatorname{CLASS}(f)=\operatorname{CLASS}(g)] \equiv \forall \alpha \in N^{\prime}[f(\alpha)=g(\alpha)} \\
\text { or } f \text { and } g \text { are both undefined for } \alpha]
\end{gathered}
$$

To see how this works, consider the partial assignment functions specified in (6).

(6) a. $f_{a}=\left\{\left\langle x^{\prime}, w_{1}\right\rangle,\left\langle y, d_{1}\right\rangle\right\}$

b. $f_{b}=\left\{\left\langle x^{\prime}, w_{1}\right\rangle,\left\langle y, d_{2}\right\rangle\right\}$

c. $f_{c}=\left\{\left\langle x^{\prime}, w_{1}\right\rangle,\left\langle y, d_{3}\right\rangle\right\}$

d. $f_{d}=\left\{\left\langle x^{\prime}, w_{2}\right\rangle,\left\langle y, d_{4}\right\rangle\right\}$

e. $f_{e}=\left\{\left\langle x^{\prime}, w_{2}\right\rangle,\left\langle y, d_{5}\right\rangle\right\}$

Since we will be needing to examine many sets of assignment functions in what follows, it will be more convenient to convey the information displayed in (6) as presented in the table in (7).$$
x^{\prime} \quad y
$$
a. $w_{1} d_{1}$
b. $w_{1} d_{2}$
c. $w_{1} d_{3}$
d. $w_{2} \quad d_{4}$
e. $w_{2} d_{5}$

The assignment functions given in (a), (b), and (c) are members of the same equivalence class, since they all assign the one primed variable to the same entity, namely, $w_{1}$. Similarly, the assignment functions specified in (d) and (e) constitute a second equivalence class.

The truth conditions of adverbial quantifiers, then, will depend on equivalence classes of assignment functions, rather than directly on individual assignment functions. In particular, the truth conditions of usually are given in (8).

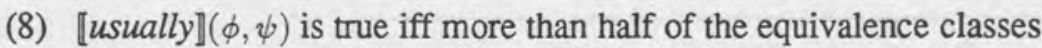
which contain an assignment function verifying $\phi$ also contain an assignment function verifying $[\phi \wedge \psi]$.

Two examples will illustrate how these rules predict a truth value for a sentence construed under a particular proportional reading when evaluated against a specific set of facts. 
(9) Usually, if a woman owns a dog, she is happy.

We have seen that (9) favors a woman-dominant asymmetric reading, so we prime the woman variable but not the dog variable in the translations of the restriction $\phi$ and the nuclear scope $\psi$ :

(10) a. $\phi=\left[\operatorname{woman}\left(x^{\prime}\right) \wedge \operatorname{dog}(y) \wedge \operatorname{owns}\left(x^{\prime}, y\right)\right]$

b. $\psi=\left[\operatorname{happy}\left(x^{\prime}\right)\right]$

Now consider the facts reported in (11).

$$
x^{\prime} \quad y \quad \text { verifies } \phi ? \text { verifies }[\phi \wedge \psi] \text { ? }
$$

a. $w_{1} d_{1}$ yes yes

\begin{tabular}{lllll}
\hline b. & $w_{2}$ & $d_{2}$ & yes & yes \\
\hline c. & $w_{3}$ & $d_{3}$ & yes & no \\
d. & $w_{3}$ & $d_{4}$ & yes & no \\
e. & $w_{3}$ & $d_{5}$ & yes & no
\end{tabular}

Since $x^{\prime}$ is the only primed variable, we have three quantificational cases, one for each woman. The third case, the one corresponding to woman $w_{3}$, contains three distinct assignment functions, one for each dog she owns. The first two cases verify the nuclear scope as well as the restriction, so they confirm the generalization expressed by (9); the third case, however, contains no assignment function which simultaneously verifies both the restriction and the nuclear scope. Nevertheless, two out of three cases confirm the generalization, and the sentence is correctly predicted to be true in this situation under the specified reading.

(12) Usually, if a woman owns a donkey, she deducts it from her taxes.

This sentence favors a symmetric interpretation, so we prime both variables:
a. $\phi=\left[\operatorname{woman}\left(x^{\prime}\right) \wedge \operatorname{donkey}\left(y^{\prime}\right) \wedge \operatorname{owns}\left(x^{\prime}, y^{\prime}\right)\right]$
b. $\psi=\left[\operatorname{deducts}\left(x^{\prime}, y^{\prime}\right)\right]$ $x^{\prime} \quad y^{\prime} \quad$ verifies $\phi$ ? verifies $[\phi \wedge \psi]$ ?

a. $w_{1} d_{1}$ yes yes

\begin{tabular}{lllll}
\hline b. & $w_{2}$ & $d_{2}$ & yes & yes \\
\hline c. & $w_{3}$ & $d_{3}$ & yes & no \\
\hline d. & $w_{3}$ & $d_{4}$ & yes & no \\
\hline e. & $w_{3}$ & $d_{5}$ & yes & no
\end{tabular}


Here we have women owning donkeys instead of dogs, but the pattern of facts is exactly the same as for the previous example, as can be seen by comparing the last column of (14) with that of (11). The difference here is that both variables are primed, so that two assignment functions will be members of the same case only if they agree both on what they assign to the woman variable and to the donkey variable. This means in effect that each assignment function constitutes a separate case. The result is that now only two out of five cases confirm the generalization, so the sentence is predicted false in this situation on the symmetric reading. Thus the same pattern of facts can either verify or falsify a proportional sentence, depending on which proportional reading it is construed under, i.e., depending on which variables get primed.

We can now restate the central problem addressed by this paper as follows: what are the structural or pragmatic factors which predict whether or not a variable can be primed?

\section{Presuppositions for proportional adverbial quantifiers}

The analysis I would like to develop depends on the hypothesis stated informally as follows: a variable will be primed only if the value of that variable potentially can determine the value of the nuclear scope independently of the values of the other variables. That is, it will be appropriate to use a primed variable only if that variable can at least potentially affect the outcome of the evaluation of the instance of quantification-there must be some correlation, causal or otherwise, between the identity of the primed variable and the value of the nuclear scope.

This hypothesis explains why (15) favors a symmetric interpretation when evaluated against facts like those in (17).

(15) Usually, if a semanticist hears of a good job, she applies for it.

(16) a. $\phi=\left[\operatorname{semanticist}\left(x^{\prime}\right) \wedge \operatorname{good}-\right.$ job $\left(y^{\prime}\right) \wedge$ hears-of $\left.\left(x^{\prime}, y^{\prime}\right)\right]$

b. $\psi=\left[\right.$ applies-for $\left.\left(x^{\prime}, y^{\prime}\right)\right]$

$\begin{array}{lllll} & x^{\prime} & y^{\prime} & \text { verifies } \phi ? & \text { veri } \\ \text { a. } & s_{1} & j_{1} & \text { yes } & \text { yes } \\ \text { b. } & s_{1} & j_{2} & \text { yes } & \text { no } \\ \text { c. } & s_{2} & j_{1} & \text { yes } & \text { no } \\ \text { d. } & s_{2} & j_{2} & \text { yes } & \text { yes } \\ \text { e. } & s_{2} & j_{3} & \text { yes } & \text { yes }\end{array}$

Considering first assignment functions $f_{a}$ and $f_{b}$, we see that semanticist $s_{1}$ applies for one job but not the other, so the value of the nuclear scope clearly depends on the identity of the job variable; hence, the job variable must be 
primed. Similarly, assignment functions $f_{a}$ and $f_{c}$ show that one semanticist applies for job $j_{1}$, but the other semanticist does not, so the semanticist variable must also be primed. This suggests that (15) should prefer a symmetric interpretation, which agrees with intuitions.

We can characterize the proposed connection between primed variables and the context by means of the formal condition defined in (18).

(18) Homogeneity:

A use of a proportional quantifier with restriction $\phi$ and nuclear scope $\psi$ satisfies the HOMOGENEITY CONDITION iff any pair of assignment functions $f$ and $g$ such that
a. $f$ and $g$ verify the restriction $\phi$ and
b. $f$ and $g$ are members of the same case

also satisfy the following:

$$
\text { c. } \llbracket \psi \rrbracket^{f}=\llbracket \psi \rrbracket^{g}
$$

This criterion boils down to the requirement that either all members of a quantificational case must agree on verifying the nuclear scope, or they all must falsify it.

The homogeneity criterion leads to predictions concerning the availability of proportional readings in specific contexts under the assumption stated in (19).

(19) Homogeneity Presupposition:

A use of a proportional adverbial quantifier when construed under a particular proportional reading is felicitous only in a context which is consistent with the homogeneity condition.

In other words, I am suggesting that the use of a proportional adverbial quantifier presupposes that the homogeneity condition is satisfied. Since different proportional readings give rise to distinct homogeneity presuppositions, only some readings will be consistent with a given context. ${ }^{1}$

1 Note that (19) as stated applies only to adverbial quantifiers. It may turn out that nominal quantifiers do not give rise to homogeneity presuppositions; this would be natural enough, given that proportional nominal quantifiers also do not give rise to distinct sets of truth conditions in the way that adverbial quantifiers can. The issues involved are complex, and a thorough evaluation would take us far away from the main purpose of this paper, which is to seek an explanation for the observed preferences for one proportional reading over another. Therefore I will leave this question for another time, though see Barker (1993) for some relevant discussion. 
Thus the homogeneity presuppositions for the preferred readings of the basic examples in (1) are: that a woman is happy with respect to all of her dogs, or to none of them; that a town is pretty or not no matter which artists live in it; but that whether a semanticist applies for a job or not depends both on the identity of the semanticist and on the identity of the job in question. These assumptions are all relatively natural, which shows that the preferred readings of these sentences are at least consistent with the homogeneity hypothesis. The remaining sections develop the empirical predictions of the hypothesis in more interesting cases.

\section{The donkey pronoun rule}

There have been a number of suggestions in the literature for predicting the availability of proportional readings. Baeurle and Egli (1985) propose the rule of thumb given in (20).

(20) Baeurle and Egli (1985): The donkey pronoun rule

A variable is more likely to be primed if there is a donkey pronoun in the nuclear scope which translates as that variable.

Note that this criterion depends only on structural properties of a sentence, and does not consider context at all. This generalization makes good predictions in many cases, including the examples given in (1) and repeated here:

(21) a. Usually, if a woman owns a dog, she is happy.

b. Usually, if an artist lives in a town, it is pretty.

c. Usually, if a semanticist hears of a good job, she applies for it.

Exactly those indefinites in the restriction which give rise to primed variables (under the preferred reading as described above) also serve as the antecedent of a donkey pronoun in the nuclear scope.

However, despite its success with some examples, the donkey pronoun rule sometimes makes exactly the wrong prediction.

(22) Usually, if a story pleases a child, it must be read over and over.

Here the only donkey pronoun in the nuclear scope takes the story indefinite as its antecedent. We would naturally expect, therefore, that this sentence should prefer a story-dominant asymmetric reading. But such a reading would predict that this sentence could be truly asserted even if most situations in which a story pleases a child do not result in repeated readings, so long as the single readings are distributed over a minority of the childpleasing stories. Judging from informants, this seems to be a highly unlikely reading for (22). On the homogeneity hypothesis, this fact is explained by observing that such a reading would presuppose that a given story will either 
be read repeatedly to every child it pleases or to none of them. In a neutral context, it is more plausible to assume that whether a story is repeated depends on the identity of the child involved as well as on the story.

In addition to (22), many of the examples in the remainder of the paper will also be cases in which the donkey pronoun rule makes inappropriate predictions.

Furthermore, note that if a nuclear scope happens not to contain a donkey pronoun, then the donkey pronoun rule fails to make any prediction at all. Nevertheless, such sentences seem just as likely to prefer one proportional reading over another.

(23) Usually, if a man opens an umbrella, it is raining.

There are no donkey pronouns in this sentence. The symmetric reading seems to be the preferred interpretation, and a man-dominant asymmetric reading may also be possible. In any case, an umbrella-dominant asymmetric reading is out of the question. That is, (23) cannot normally be interpreted as asserting that most umbrellas have the property that they are only opened when it is raining. Such a reading would be predicted to be satisfied even if the majority of men compulsively opened umbrellas on sunny days over and over again, so long as only a minority of umbrellas were involved.

The absence of an umbrella-dominant asymmetric reading is exactly what the homogeneity hypothesis would predict. Such a reading would presuppose that some umbrellas would always be opened only in the rain, while other umbrellas would always be opened only when it is not raining, no matter who is carrying them. Since this is not a very plausible assumption in a neutral context, the homogeneity hypothesis predicts that (23) will not normally have such a reading.

But why is the donkey pronoun rule so often correct? Obviously, the presence of a donkey pronoun in the nuclear scope makes it more likely that the variable associated with that pronoun will have a strong effect on the truth conditions of the nuclear scope. That is, if the nuclear scope of the sentence Usually, if a woman owns a dog, she is happy amounts to the proposition that $x$ is happy, where $x$ is the woman variable, then it is no wonder that this variable tums out to have a strong effect on whether the nuclear scope is satisfied or not.

Thus the homogeneity hypothesis is capable of explaining the insight embodied in the donkey pronoun rule while still being capable of making correct predictions in situations in which the donkey pronoun rule gives incorrect predictions or fails to make any predictions at all. 


\section{Uniqueness presuppositions}

Kadmon $(1987,1990)$ suggests that proportional readings can give rise to distinct presuppositions under certain conditions. The presuppositions she has in mind involve implications of uniqueness or relative uniqueness.
a. Usually, if a semanticist hears of a good job, she applies for it.
b. Usually, if a woman owns a dog, she talks to it.

As we have seen, the sentence in (24a) favors a symmetric interpretation, and Kadmon notes that there are no uniqueness implications for either of the variables involved. That is, (24a) can be felicitously uttered in a context in which each semanticist hears of more than one job (the referent of the job variable is not unique relative to the choice of semanticist), and in which more than one semanticist hears of each job (the referent of the semanticist variable is not necessarily unique relative to the choice of job). In contrast, (24b) favors a woman-dominant asymmetric reading, and Kadmon claims that there is a uniqueness implication, and that (24b) will be felicitous only under the assumption that there is a unique dog per woman.

In general, Kadmon (1990:301) asserts that symmetric readings never give rise to any uniqueness presuppositions, but asymmetric readings can: if the referent of an unprimed variable is referred to by a definite NP such as a donkey pronoun, then, as a result of the definiteness associated with that $\mathrm{NP}$, the referent of the variable must be unique relative to the choice of the primed variables (see Kadmon (1990:310)).

However, as noted by Kadmon, there are important exceptions to this claim, including Heim's famous sage plant sentence, a variant of which appears in (25).

(25) Usually, if a woman buys a sage plant here, she buys two others along with it.

Clearly (25) strongly prefers a woman-dominant asymmetric reading, so that the presence of the donkey pronoun it should give rise to the presupposition that the referent of the sage plant variable is unique relative to the choice of a woman. However, there is no implication that any of the sage plants is distinguished in any way from the others.

Kadmon (1990:317) offers the following rationale: if it can't possibly matter which sage plant we pick, then the non-primed variable is excused from uniqueness presuppositions. 
(26) $x^{\prime} \quad y \quad x^{\prime}$ buys two others along with $y$ ?
a. $w_{1} s_{1}$ yes
b. $w_{1} s_{2}$ yes
c. $w_{1} s_{3}$ yes
d. $w_{2} \quad s_{4}$ yes
e. $w_{2} \quad s_{5}$ yes
f. $w_{2} s_{6}$ yes
g. $\begin{array}{llll}w_{3} & s_{7} & \text { no }\end{array}$

h. $w_{3} \quad s_{8}$ no

i. $w_{4} \quad s_{9}$ no

j. $w_{5} \quad s_{10}$ no

That is, as can be seen by inspecting (26), for any choice of a woman, it doesn't matter which sage plant we pick as the referent of the pronoun: either is is true of all of them that two others were bought along with them, or or none of them.

Note that Kadmon's requirement is logically equivalent to the homogeneity presupposition for the relevant proportional reading, and furthermore that the meaning of the sage plant sentence entails homogeneity. However, there are examples similar to sage plant sentences but in which homogeneity is contingent on the context.

(27) Usually, if a person knows a symphony well, she can't help

humming along with it when she hears it on the radio.

Clearly, this sentence has an asymmetric reading on which it can't be falsified by the existence of a single woman who knows dozens of symphonies but never hums. Therefore, thanks to the definiteness of the donkey pronouns in the nuclear scope, Kadmon predicts that there should be a unique symphony per person. However, a use of (27) does not presuppose that the relevant people know at most one symphony, or that people who know more than one symphony somehow fall outside the scope of the asserted generalization.

It is not clear that Kadmon's sage plant exception applies in this situation, since it is perfectly possible to imagine that a person will always hum along with the theme to Beetovan's 9th symphony, but never with that of the 7th. Thus it 'matters' which symphony we have in mind. However, we could invoke the sage-plant exception if we first assume the homogeneity hypothesis, which predicts that specified reading is felicitous only under the assumption that each woman either hums along with all of the symphonies she knows or none of them. 
As far as I know, the homogeneity hypothesis is consistent with $\mathrm{Kad}$ mon's claims about the uniqueness presuppositions associated with proportional readings, and in fact, as we have just seen, homogeneity may be crucially involved in order for Kadmon's theory to make the correct prediction for certain examples such as (27). It is worth noting that sage plant sentences, far from being systematic exceptions to the general theory, as they are for Kadmon, are prototypical examples confirming the predictions of the homogeneity hypothesis.

In any case, Kadmon's theory without elaboration cannot provide a complete theory of the availability of proportional readings in context, as shown by (27). Furthermore, just as for the donkey pronoun rule discussed in the previous section, Kadmon's theory fails to make any predictions at all in the absence of donkey pronouns or definite descriptions in the nuclear scope. Therefore the homogeneity hypothesis is motivated independently of Kadmon's theory.

\section{Explicit and implicit focus structures}

Kadmon (1987), Heim (1990), and others have observed that focus seems to affect the availability of proportional readings.

(28) a. Usually, if a drummer lives in an apartment building, it is half empty.

b. Usually, if a drummer lives in an APARTMENT BUILDING, it is half empty.

For instance, the sentence in (28a) normally prefers an object-dominant asymmetric reading. Thus a single full apartment building which houses most of the drummers is not sufficient to falsify (28a). However, if the apartment building description receives contrastive stress, as in (28b), then a drummer-dominant asymmetric reading or a symmetric reading becomes more prominent. For these readings, it is the housing preferences of individual drummers that we are concerned with, and if a number of drummers live in the same apartment building, they can each constitute a separate counterexample to the generalization expressed by the quantificational token, so that a single apartment building full of drummers can falsify (28b). (29).

Contrasts like the one in (28) suggest the descriptive generalization in

(29) Variables in focus don't get primed.

Krifka (1992) proposes a mechanism which accounts for this generalization. As part of a general theory of the interaction of focus with quantification, he 
proposes that if clauses must routinely be factored into a focus and a quantificational background. In effect, his framework guarantees that variables in focus undergo existential quantification, and variables in the quantificational background are bound by the quantifier, giving truth conditions, which, as near as I can tell, are compatible with the ones given here. Thus Krifka suggest that predicting which variables are primed is just a special case of the more general problem of deciding which NPs are in semantic focus.

One difficulty with evaluating this hypothesis is that it is not always easy to tell which elements of a sentence are in focus, especially in the absence of any marked degree of intonational stress. However, following Krifka, we can assume that if an NP does receive contrastive intonational stress, then either it or some constituent containing it is in focus. Our strategy for testing (29), then, will be to look for situations in which a description receives contrastive stress but which nevertheless must be primed.

To further develop the drummer example, imagine that we are talking about the housing preferences of musicians. Most musicians usually prefer to live in isolated houses so that they can practice late at night without disturbing their neighbors. Therefore, if a musician lives in an apartment building instead, there must be some overriding advantage. For instance, perhaps cello players, bassoon players, and tuba players will live in an apartment building if it is sufficiently close to the conservatory practice rooms, since that means they won't have to carry their heavy instruments as far. In this context, consider a token of (30).

(30) Usually, if a DRUMMER lives in an apartment building, it is close to the bars downtown.

It seem to me that in this context, (30) can have a drummer-dominant asymmetric reading (and perhaps also a symmetric reading). To see this, note that (30) has a reading which can be verified merely if most of the drummers live in a single large apartment building downtown, even if a smaller number of drummers live in multiple apartment buildings closer to the conservatory. Such truth conditions result only when the drummer variable is primed.

Note also that the availability of such a reading is consistent with the homogeneity hypothesis, which requires that for any given drummer, when she chooses to live in an apartment building, she will either always choose one that is downtown, or she never will, which is a plausible assumption in the specified context. (1989b):

A similar type of example is discussed in some detail in Kratzer

(31) If a SiclLIAN adores a piece of music, it is rarely a Bellini opera. 
Kratzer observes that a piece-of-music asymmetric reading is completely impossible. That is, there is no reading of (31) which depends only on the number of Sicilian-liked pieces of music which happen to be Bellini operas. If there were, (33) could still be true even if there were thousands of Sicilians who adored Bellini operas, so long as there were a few Sicilians who like enough Mozart and Verdi operas to outnumber Bellini's oeuvre.

The existence of examples like (30) and (31) motivates the observation stated in (32).

(32) Sometimes an indefinite which receives contrastive stress gives rise to a variable which must be primed.

On the face of it, this fact at least calls into question the assumption that being in focus is incompatible with translating as a primed variable.

How serious of a problem is this for a focus-based theory of proportional ambiguity? There may be a way out. Krifka (1992, p.c.) makes the point that there may be more than one focus structure involved in such examples. There may be one focus structure for the if clause, and a second focus structure for an implicit discourse-level focus operator associated with the sentence as a whole. In such cases of embedded focus, sentence accent aligns with the highest operator. It is possible, then, that the contrastive stress on drummer in (30) only reflects the fact that the drummer description is focussed at the level of the sentence as a whole, at the same time that the drummer indefinite is not in focus with respect to the local if clause. If so, then Krifka's theory at least doesn't make any wrong predictions with respect to these examples.

An embedded-focus story may turn out to be correct for these examples. If so, where does this leave us? To the extent that surface marking underdetermines the focus structure hypothesized by Krifka's theory, we are still faced with situations in which we have no explanation for why some variables can be in focus and some cannot be. To the extent that the homogeneity hypothesis is capable of making good predictions in such cases, it is motivated independently of the semantics of focus.

\section{Weak readings and domain narrowing}

One potential threat to the generality of the homogeneity hypothesis comes from the so-called weak versus strong interpretations for some quantificational sentences as discussed by Heim (1982:61-2), Schubert and Pelletier (1989), Gawron, Nerbonne and Peters (1992), and Chierchia (1992), among others.

(33) Usually, if a man has a quarter in his pocket, he puts it in the meter. 
The sentence in (33) favors an asymmetric reading on which there is one case per man. Thus the homogeneity thesis would seem to predict that a use of (33) on this reading will presuppose that each man will either put all or none of his quarters in the meter. But a use of (33) seems to be perfectly felicitous in a context in which each man puts only as many quarters in the meter as he needs to: some quarters go into the meter, and some will remain in the man's pocket. Are homogeneity presuppositions somehow suspended for this example?

Gawron, Nerbonne and Peters (1991) and Chierchia (1992) suggest that strong readings arise from the presence of E-type pronouns. In essence, they restrict Kadmon's explanation for the failure of uniqueness for donkey pronouns in sage-plant examples so as to apply specifically to pronouns with E-type denotations: a use of an E-type pronoun will be felicitous in a context in which multiple entities satisfy the implicit descriptive content of the pronoun only if the choice of such an entity is immaterial to the outcome of the quantification. I see two empirical problems with this strategy. First, strong readings seem to be possible even without the presence of E-type pronouns.

(34) a. Usually, if a man saw a truck coming, he got out of the way.

b. Most men who saw a truck coming got out of the way.

Both sentences in (34) entail that each man confirming the generalization got out of the way of all of the trucks he saw coming.

The second problem is that this account makes incorrect predictions in other contexts arguably involving E-type pronouns, such as paycheck sentences.

(35) a. The man who sent his grandmother to a nursing home was kinder than the man who threw her out on the street.

b. The woman who put her hand on the fridge was luckier than the woman who put it on the stove.

If the paycheck pronouns her and it are indeed E-type pronouns, then we should expect that (35a) presupposes that the second man threw both of his grandmothers out on the street, and that (35b) presupposes that the second woman put both of her hands on the stove. But these sentences give rise to no such presuppositions.

I would like to suggest instead that weak readings are just a special case of the independently motivated mechanism of domain narrowing. Once contextual domain narrowing is taken into account, examples such as (33) can be seen to behave exactly as expected and require no weakening of the homogeneity hypothesis.

For nominal quantification, domain narrowing explains why, for example, a universal quantifier such as every can be true in the face of apparent 
counterexamples. Thus the reason that Every tree is laden with wonderful apples is not almost invariably false is that it can be understood as if it applied only to the trees within the bounds of a certain contextually salient orchard (see Kratzer (1989a) or Roberts (to appear)). For nominal quantification, then, domain narrowing allows for quantification over a contextually restricted set of individuals. But adverbial quantifiers do not quantify over individuals; roughly speaking, they quantify over situations (or partial situations), as approximated in this paper as partial assignment functions. Therefore domain narrowing for adverbial quantification would involve quantification over a restricted set of assignment functions.

In order to see which assignment functions are indeed relevant for the parking situation, we must be more explicit about the assumptions implicit in the context. Do we need to consider every situation involving a man and a parking meter? Clearly not. A number of ancillary propositions must hold: the man must have just parked his car in front of the parking meter in question, it must be the law of the land that during certain hours of the day the meter must be fed, the man must be aware of these laws, the meter in question must not have time left on it from the last driver, and so on. Basically, a parking situation will be relevant for deciding the truth of (33) only if it is also a situation in which the meter needs to be fed.

Now consider a specific context in which homogeneity seems to be violated.

$$
x^{\prime} \quad y \quad x^{\prime} \text { put } y \text { in the meter? }
$$

a. $m_{1} \quad q_{1}$ yes

b. $m_{1} \quad q_{2}$ no

c. $m_{1} \quad q_{3}$ no

d. $m_{2} \quad q_{4}$ yes

e. $m_{2} \quad q_{5}$ yes

In (36), man $m_{1}$ puts quarter $q_{1}$ into the meter, and leaves quarters $q_{2}$ and $q_{3}$ in his pocket. The assignment functions in (36b) and (36c), then, are the ones which seem to violate homogeneity. However, these assignment functions are not relevant for deciding the truth of (33), since they correspond to situations in which the man has already put a quarter into the machine. They are no more relevant than situations in which, say, the meter is broken, or in which it is the middle of the night (when parking laws don't apply). Once we restrict the domain of quantification to exclude such assignment functions, the resulting set of assignment functions satisfies homogeneity.

One particularly striking way to see the importance of the contextually supplied implicit assumptions that this explanation relies on is to see what 
happens when the supposedly crucial assumption is not present. Imagine that we are talking instead about the habits of gamblers.

(37) Usually, if a man has a quarter in his pocket, he puts it in the slot.

In this situation, the implicit assumptions are roughly parallel, except that there is no longer any assumption that the slot machine will change state (in any relevant way) after the insertion of each quarter. Therefore a situation in which a man has just put a quarter into the slot of a slot machine is just as much an opportunity to gamble as before. Thus we correctly predict that (37) does not seem to have a so-called weak interpretation parallel to that alleged for (33). That is, (37) cannot be verified merely if each man puts a single quarter in the slot machine. Here all of the assignment functions in (36) are equally relevant, and the force of the homogeneity presupposition comes through exactly as predicted.

\section{Summary}

We have seen that a sentence involving a proportional adverbial quantifier is ambiguous across a number of readings which have distinct truth conditions. These readings are characterized by the status of the indefinites in the restriction of the quantifier, i.e., whether or not they translate as primed variables. However, only some readings will be felicitous in a given context. More specifically, the context must be consistent with the homogeneity presupposition induced by the reading in question, which requires that all members of a case must agree on whether they satisfy the nuclear scope. This homogeneity hypothesis explains why the distribution of donkey pronouns in the nuclear scope is such a good indicator of the preferred proportional reading in general, but it also makes correct predictions where the donkey-pronoun rule makes incorrect predictions. The homogeneity hypothesis is also consistent with the relevant predictions of Kadmon's theory of uniqueness presuppositions for asymmetric quantification. In particular, sage plant contexts are prototypical examples of situations which satisfy the homogeneity criterion. Furthermore, the homogeneity hypothesis makes good predictions in some situations in which neither the donkey-pronoun theory nor Kadmon's uniqueness theory make any predictions at all, namely, sentences in which the nuclear scope does not contain any donkey pronouns (or other definite descriptions that denote one of the variables introduced by the restriction). The homogeneity hypothesis is also consistent with Krifka's theory of the connection between focus structure and semantic interpretation, and it makes good predictions in situations in which the relevant details of the focus structure are at best underdetermined. 


\section{References}

Barker, Chris (1993) 'Unselective Determiners', to appear in the proceedings of WCCFL XII.

Baeuerle, R. and U. Egli (1985) 'Anapher, Nominalphrase und Eselssaetze', Papier 105 des Sonderforschungsbereichs 99, Universitaet Konstanz. Chierchia, Gennaro (1992) 'Anaphora and Dynamic Binding', Linguistics and Philosophy 15.2: 111-183.

Gawron, Jean Mark, John Nerbonne, and Stanley Peters (1991) 'The absorption principle and E-type anaphora', in Jon Barwise et al. (eds.), Situation Theory and Its Applications, volume 2, Center for the Study of Language and Information, Stanford.

Heim, Irene (1982) The Semantics of Definite and Indefinite Noun Phrases, dissertation, University of Massachusetts, Amherst.

Heim, Irene (1990) 'E-Type Pronouns and Donkey Anaphora', Linguistics and Philosophy 13: 137-177.

Kadmon, Nirit (1987) On Unique and Non-unique Reference and Asymmetric Quantification, $\mathrm{PhD}$ dissertation, University of Massachusetts, Amherst.

Kadmon, Nirit (1990) 'Uniqueness', Linguistics and Philosophy 13:273324.

Kratzer, Angelika (1989a) 'An Investigation of the Lumps of Thought', Linguistics and Philosophy 12.5:607-653.

Kratzer, Angelika (1989b) 'Stage-level and individual level predicates', ms., University of Massachusetts, Amherst.

Krifka, Manfred (1992) 'A framework for focus-sensitive quantification', in C. Barker and D. Dowty (eds.) Proceedings of the Second Conference on Semantics and Linguistic Theory, Ohio State University Working Papers in Linguistics volume 40, Department of Linguistics, Ohio State University, Columbus, 215-236.

Lewis, David (1975) 'Adverbs of Quantification', in E. Keenan, ed., Formal Semantics, Cambridge University Press, Cambridge, UK.

Partee, Barbara (1984) 'Nominal and temporal anaphora', Linguistics and Philosophy 7: 243-286.

Roberts, Craige (to appear) 'Domain selection in dynamic semantics', in Emmon Bach, Eloise Jelinek, Angelika Kratzer, and Barbara H. Partee (eds.), Cross-Linguistic Quantification, Kluwer, Dordrecht.

Root, R. (1985) The Semantics of Anaphora in Discourse, PhD dissertation, University of Texas, Austin.

Schubert, Lenhart K. and Francis Jeffrey Pelletier (1989) 'Generically speaking', in Gennaro Chierchia, Barbara H. Partee and Raymond Turner 
(eds.), Properties, Types and Meaning, volume II: Semantic Issues, 193-268.

de Swart, H. (1991) Adverbs of Quantification: a Generalized Quantifier Approach, $\mathrm{PhD}$ dissertation, Groningen.

Chris Barker

Center for Cognitive Science

208 Ohio Stadium East

1961 Tuttle Park Place

Columbus, $\mathrm{OH} 43210$

barker@ling.ohio-state.edu 